\title{
Progress in Chemical Constituents and Pharmacological Effects of Citrus medica L. var. sarcodactylis Swingle
}

\author{
Shuyu Xu, Yin Qu, Xinyu Liu, Yao Li, Jimin Liu, and Hezhong Jiang* \\ School of Life Science and Engineering, Southwest Jiaotong University, Chengdu, Sichuan 611756, China \\ Email: jianghz10@swjtu.edu.cn (H. J.)
}

\begin{abstract}
Citrus medica L. var. sarcodactylis Swingle is known as Foshou fruit that can be used as Chinese medicinal materials, and it has a health and longevity effect. In recent years, investigations about the chemical compounds of Citrus medica L. var. sarcodactylis Swingle have shown that its main compounds include flavonoids, essential oils, coumarins, and polysaccharides, with anti-inflammatory, antimicrobial, antioxidant activities, an effect on the autonomic nervous system, and other pharmacological effects. In this paper, the chemical compositions and pharmacological effects of Citrus medica L. var. sarcodactylis Swingle are summarized.
\end{abstract}

Keywords Citrus medica L. var. sarcodactylis Swingle, chemical constituents, pharmacological effects

\section{Introduction}

Citrus medica L. var. sarcodactylis Swingle (Rutaceae), one of the variants of Citrus medica L. var. medica, is an evergreen shrub or small tree and widely cultivated in Zhejiang, Fujian, Guangdong, Guangxi, Yunnan and Sichuan Province of China. Citrus medica L. var. sarcodactylis Swingle has the function of protecting liver, stomach and pancreas, and can treat hypertension and respiratory tract infection. ${ }^{[1]}$ In addition, it is traditionally used for the treatment of hypertension, respiratory tract infections and tracheitis in China. ${ }^{[2]}$ Due to the above effects, Citrus medica L. var. sarcodactylis Swingle has gradually attracted widespread attention in recent years. Herein, we summarize the chemical constituents and pharmacological effects of Citrus medica L. var. sarcodactylis Swingle.

\section{Chemical Constituents of Citrus medica L. var. sarcodactylis Swingle}

The chemical compositions of Citrus medica L. var. sarcodactylis Swingle are reported mainly including phenolic components, terpenoids, essential oil, and other components. ${ }^{[3]}$

\section{Phenolic constituent}

The phenolic components of Citrus medica L. var. sarcodactylis Swingle are mainly flavonoids, coumarins, and other phenolic components (Figure 1).

The flavonoids are the main components in Citrus medica $\mathrm{L}$. var. sarcodactylis Swingle. In 1984, He and Ling isolated two flavonoids from Citrus medica L. var. sarcodactylis Swingle, named 3,5,6-trihydroxy-4',7-dimethoxyflavone (1) and 3,5,6-trihydroxy-3',4',7'-trimethoxyflavone (2). ${ }^{[4]}$ Feng et al. isolated diosmetin (3), and diosmin (4) from the ethanol extract of Citrus medica L. var. sarcodactylis Swingle in 2004..$^{[5]}$ In 2010, Chan et al. used a combination of traditional chromatography techniques isolated atalantoflavon (5), lonchocarpol A (6), and C-glycosylflavone-vitexin (7). ${ }^{[6]}$

Coumarins are also important phenolic compounds of Citrus medica L. var. sarcodactylis Swingle. Gao et al. isolated 5,7-dimethoxycoumarin (8) from Citrus medica L. var. sarcodactylis Swingle in 2002. ${ }^{[7]}$ In 2010, Chan et al. purified a new coumarin named citrumedin-B (9) and xanthyletin (10),
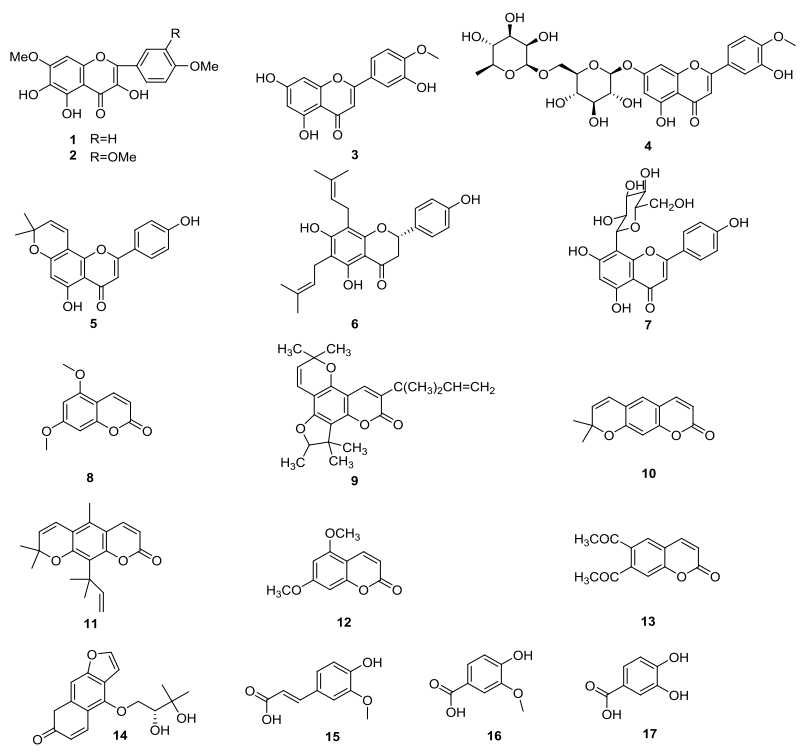

10
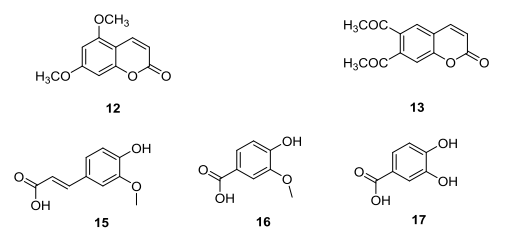

13

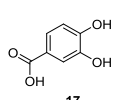

Figure 1 The structures of phenolic components isolated from Citrus medica L. var. sarcodactylis Swingle.

nordentatin (11), 5,7-dimethoxycoumarin (12) ${ }^{[6]}$ In 2017, Chan et al. found 6,7-dimethoxycoumarin (13) from Citrus medica L. var. sarcodactylis Swingle. They proved that it has a significant inhibitory effect on superoxide anion formation. ${ }^{[8]}$

In 2004, Feng et al. isolated aviprin (14), ferulic acid (15), vanillic acid (16), and protocatechuic acid (17) from the ethanol extract of Citrus medica L. var. sarcodactylis Swingle. ${ }^{[5]}$

\section{Terpenoids}

The terpenoids in Citrus medica L. var. sarcodactylis Swingle are mainly triterpenoids especially tetranortriterpenoids, and sesquiterpenoids.

In 2010, Chan et al. purified thirty known compounds from Citrus medica L. var. sarcodactylis Swingle by using a combination of traditional chromatography techniques, including $1-(10 \rightarrow 19)$ abeo-7 $\alpha$-acetoxy-10 $\beta$-hydroxyisoobacunoic acid-3,10-lactone (18), nomilin (19), limonin (20), lupeol 


\section{Minireview}

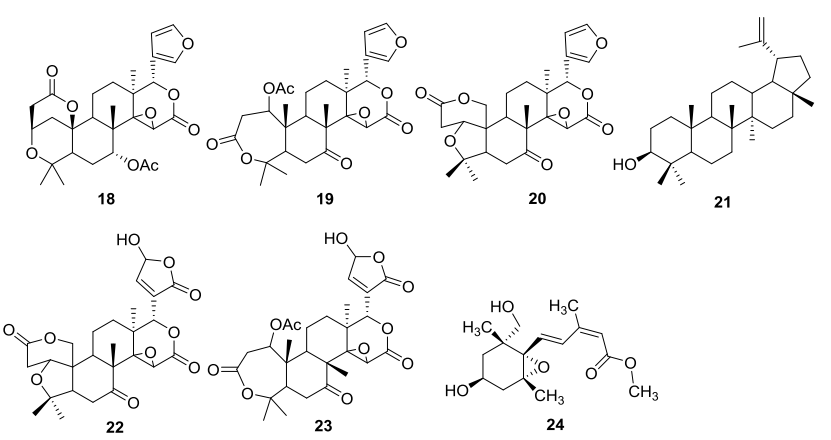

Figure 2 The structures of terpenoids isolated from Citrus medica L. var. sarcodactylis Swingle.

(21), limonexic acid (22), citrusin (23) (Figure 2). ${ }^{[6]}$

Then in 2017, Chan et al. found a new sesquiterpene named citrumedin- $C$ (24) by investigation of the chemical constituents from the fruits of Citrus medica L. var. sarcodactylis Swingle. ${ }^{[8]}$

\section{Essential oil}

Essential oil is an important part of the study of Citrus medica L. var. sarcodactylis Swingle. Kim et al. studied the composition of essential oil of Citrus medica L. var. sarcodactylis Swingle by GC-MS, and identified fifteen components in $2013 .^{[9]}$ According to their study, the main components of the essential oil of Citrus medica L. var. sarcodactylis Swingle are limonene (25) and y-Terpinene (26) (Figure 3). Mitropoulou et al. used SPME GC-MS to analyze the main compounds of the essential oil of $C$. medica peels and assayed the antimicrobial properties in 2017. ${ }^{[10]}$ In 2018, Guo et al. compared chemical composition, antimicrobial and antioxidant activity of essential oil from Citrus medica L. var. sarcodactylis Swingle. ${ }^{[1]}$ They used GC-MS analysis for identifying more than 200 chemical components from the essential oil of Citrus medica L. var. sarcodactylis Swingle, which contained $D$-limonene (27), a-pinene (28), myrcene (29), ocimene (30), and linalool (31).

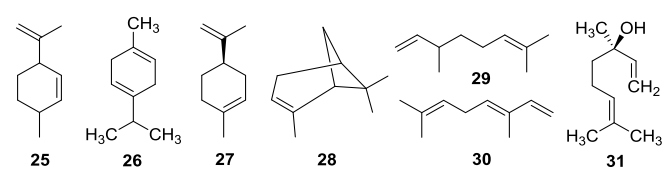

Figure 3 The structures of essential oil from Citrus medica L. var. sarcodactylis Swingle.

\section{Other compounds}

In addition to the above chemical components, Gao et al. isolated $\triangle^{5,22}$-stigmasterol (32) and palmitic acid (33) from the ethyl acetate part of its ethanol extract in 2002. It is the first time $\triangle^{5,22}$-stigmasterol was isolated from Citrus medica L. var. sarcodactylis Swingle. ${ }^{[7]}$ In 2004, Feng et al. isolated a new polysaccharide 5-methoxyfurfural and Kojic acid (34) from the ethanol extract of Citrus medica L. var. sarcodactylis Swingle. ${ }^{[5]}$

\section{Pharmacological Activities}

Citrus medica L. var. sarcodactylis Swingle is a traditional Chinese medicinal plant with a variety of pharmacological activities such as antimicrobial, anti-inflammatory, antioxidative effect, and so on. ${ }^{[12]}$

\section{Antimicrobial activity}

Theanphong et al. tested antimicrobial activity of essential oil with agar diffusion method and found its significant antimicrobial activity against $\mathrm{S}$. aureus and $\mathrm{B}$. subtilis with the same minimum inhibitory concentration of $2500 \mathrm{ppm} .{ }^{[13]} \mathrm{Li}$ et al. studied antimicrobial effect of Citrus medica $L$. var. sarcodactylis Swingle essential oil and its mechanism against food-borne bacteria. Then, they found that the essential oil showed a significant reduction effect on the growth rate of bacteria, and led to lysis of the cell wall, intracellular ingredient leakage, cell death eventually. ${ }^{[14]}$

\section{Anti-inflammatory activity}

Kim et al. showed that the essential oil of Citrus medica L. var. sarcodactylis Swingle inhibited LPS-stimulated inflammation by blocking the NF-KB, JNK, and ERK pathways in macrophages, and proved essential oil possessed antiinflammatory properties. ${ }^{[9]}$

\section{Antioxidative activity}

Zhu et al. found that the bergamot polysaccharide of Citrus medica L. var. sarcodactylis Swingle could efficiently remove $\mathrm{O}_{2}^{-}, \mathrm{HO}^{-}$and protect DNA chains significantly from being damaged by free radicals. ${ }^{[15]}$

\section{Effect on the autonomic nervous system}

Sayorwan et al. investigated the Citrus medica L. var. sarcodactylis Swingle peel oil effect on the autonomic nervous systems and mood responses after inhaling. ${ }^{[16]}$

\section{Other pharmacological activities}

Peng et al. proved that Citrus medica L. var. sarcodactylis Swingle fruit can promote insulin secretion by kinetic analysis on the hypoglycemic patterns of the intraperitoneal glucose tolerance (IPGTT) and the insulin-glucose tolerance tests (IGTT) ${ }^{[1]}$

\section{Conclusions and Perspectives}

As one traditional Chinese medicine, Citrus medica L. var. sarcodactylis Swingle is distributed widely and it has a long medicinal history. The chemical constituents of Citrus medica L. var. sarcodactylis Swingle include polysaccharide, flavonoids, essential oils, coumarins, sesquiterpene, and other compounds. Pharmacodynamic studies show that it has a variety of pharmacological effects. At present, the research on Citrus medica L. var. sarcodactylis Swingle is mainly focused on the components and functions of essential oil, its other components and bioactivities need to be further studied.

\section{Acknowledgement}

This work was co-supported by the research project of the Fund of Science and Technology Agency of Chengdu (No. 2019-YF09-00049SN), the Administration of Traditional Chinese Medicine of Sichuan (No. 2017PC004), the 13th Personalized Experimental Project (Nos. GX201913085 and GX201913100), and the Student Research Training Program (SRTP, 2019156).

\section{Author Contributions}

Following are the details of the contributions made by each of the authors for the manuscript: Hezhong Jiang and Shuyu Xu performed the data analyses and wrote the manuscript; Yin Qu and Xinyu Liu contributed to the conception of the study; Yao Li and Jimin Liu contributed to analysis and manuscript preparation.

\section{Conflict of Interest}

The authors declare no conflict of interest.

Copyright () 2020 Shuyu Xu, Yin Qu, Xinyu Liu, Yao Li, Jimin Liu, and Hezhong Jiang. This article is an open access article distributed under 
the terms and conditions of the Creative Commons Attribution (CC BY) license (http://creativecommons.org/licenses/by/4.0/). The use, distribution or reproduction in other forums is permitted, provided the original author(s) or licensor are credited and that the original publication in this journal is cited, in accordance with accepted academic practice. No use, distribution or reproduction is permitted which does not comply with these terms.

\section{References}

[1] Peng, C. H.; Ker, Y. B.; Weng, C. F. Insulin Secretagogue Bioactivity of Finger Citron Fruit (Citrus medica L. var. Sarcodactylis Hort, Rutaceae). J. Agric. Food. Chem. 2009, 57, 8812-8819.

[2] He, Z.; Liang, F.; Zhang, Y. Water-soluble polysaccharides from finger citron fruits (Citrus medica L. var. sarcodactylis). Carbohydr. Res. 2014, 388, 100-104.

[3] Nogata, Y.; Sakamoto, K.; Shiratsuchi, H. Flavonoid Composition of Fruit Tissues of Citrus Species. Biosci. Biotechnol. Biochem. 2006, 70, 178-192.

[4] He, H. Y.; Ling, L. Q. Chemical studies on a Chinese traditional drug fingered citron(Citrus medica L. var sarcodactylis (Noot.) Swingle). Acta Pharm. Sinica 1985, 6, 433-435.

[5] Yin, F.; Lou, F. Studies on the Constituents of Citrus medica L. var. Sarcodactylis (Noot.) Swingle. Chin. Pharm. J. 2004, 39, 24-26.

[6] Chan, Y. Y.; Li, C. H.; Shen, Y. C. Anti-inflammatory Principles from the Stem and Root Barks of Citrus medica. Chem. Pharm. Bull. 2010, 58, 61-65.

[7] Gao, Y. H.; Xu, H. H.; Diao, Y. M. Studies on Chemical Components of Citrus medica L. var. Sarcodactylis Swingle. Chin. Med. Mater. 2002, 13, 315-316

[8] Chan, Y. Y.; Hwang, T. L.; Kuo, P. Constituents of the Fruits of Citrus medica L. var. sarcodactylis and the Effect of 6,7-Dimethoxy-coumarin on Superoxide Anion Formation and
Elastase Release. C. Molecules 2017, 22, 1454-1463.

[9] Kim, K. N.; Ko, Y. J.; Yang, H. M. Anti-inflammatory effect of essential oil and its constituents from fingered citron (Citrus medica L. var. sarcodactylis) through blocking JNK, ERK and NF-KB signaling pathways in LPS-activated RAW 264.7 cells. Food Chem. Toxicol. 2013, 57, 126-131.

[10] Mitropoulou, G.; Fitsiou, E.; Spyridopoulou, K. Citrus medica essential oil exhibits significant antimicrobial and antiproliferative activity. Lwt-Food Sci. Technol. 2017, 84, 344-352.

[11] Guo, J. J.; Gao, Z. P.; Xia, J. L. Comparative analysis of chemical composition, antimicrobial and antioxidant activity of citrus essential oils from the main cultivated varieties in China. Lwt-Food Sci. Technol. 2018, 97, 825-839.

[12] Sakurada, T.; Kuwahata, H.; Katsuyama, S. Intraplantar injection of bergamot essential oil into the mouse hindpaw: effects on capsaicin-induced nociceptive behaviors. Int. Rev. Neurobiol. 2009, 85, 237-248.

[13] Theanphong, O.; Songsak, T.; Mingvanish, W. Chemical Composition and Antimicrobial Activity of the Essestial Oil from Citrus medica L. var. sarcodactylis (Sieber) Swingle Leaf. Mahidol Univ. J. Pharm. Sci. 2008, 35, 57-61.

[14] Li, Z. H.; Cai, M.; Liu, Y. S. Antibacterial Activity and Mechanisms of Essential Oil from Citrus medica L. var. sarcodactylis. Molecules 2019, 24, 1577-1587.

[15] Zhu, X. Y.; Wu, J. M.; Jia, Z. S. Composition and Antioxidative Actvity of Polysaccharide from Bergamot. Chem. J. Chin. Univ. 2005, 26, 1264-1267.

[16] Sayorwan, W.; Rumruay, V. Chemical compositions of fingered citron peel (Citrus medica L. var. sarcodactylis) and its effect on the autonomic nervous system. J. Health Res. 2017, 31, 307-313.

Received December 23, 2019 Accepted January 17, 2020 